\title{
Comparative study of whole exome sequencing-based copy number variation detection tools
}

\author{
Lanling Zhao', Han Liu', Xiguo Yuan², Kun Gao and Junbo Duan ${ }^{1 *}$ (D)
}

\begin{abstract}
Background: With the rapid development of whole exome sequencing (WES), an increasing number of tools are being proposed for copy number variation (CNV) detection based on this technique. However, no comprehensive guide is available for the use of these tools in clinical settings, which renders them inapplicable in practice. To resolve this problem, in this study, we evaluated the performances of four WES-based CNV tools, and established a guideline for the recommendation of a suitable tool according to the application requirements.

Results: In this study, first, we selected four WES-based CNV detection tools: CoNIFER, cn.MOPS, CNVkit and exomeCopy. Then, we evaluated their performances in terms of three aspects: sensitivity and specificity, overlapping consistency and computational costs. From this evaluation, we obtained four main results: (1) The sensitivity increases and subsequently stabilizes as the coverage or CNV size increases, while the specificity decreases. (2) CoNIFER performs better for CNV insertions than for CNV deletions, while the remaining tools exhibit the opposite trend. (3) CONIFER, Cn.MOPS and CNVkit realize satisfactory overlapping consistency, which indicates their results are trustworthy. (4) CoNIFER has the best space complexity and cn.MOPS has the best time complexity among these four tools. Finally, we established a guideline for tools' usage according to these results.

Conclusion: No available tool performs excellently under all conditions; however, some tools perform excellently in some scenarios. Users can obtain a CNV tool recommendation from our paper according to the targeted CNV size, the CNV type or computational costs of their projects, as presented in Table 1, which is helpful even for users with limited knowledge of computer science.
\end{abstract}

Keywords: Copy number variants, Next generation sequencing, Whole exome sequencing, Sensitivity, Specificity, Overlapping consistency, Computational costs, Recommendation, Guideline

\section{Background}

Copy number variation $(\mathrm{CNV})$ is a phenomenon that is caused by genomic rearrangement, and the CNV length typically exceeds 1 kilobase $(\mathrm{kb})$ [1]. In medicine, the frequency of CNVs is $12 \%$ [2]; hence, it is an important component of gene variations and plays an important role in generating the necessary variation of population and of disease phenotypes [3,4]. Since the CNV is of substantial medical significance, it has become a hotspot in current medical research, and many accurate $\mathrm{CNV}$ identification methods have been proposed in the past

\footnotetext{
* Correspondence: junbo.duan@mail.xjtu.edu.cn

'Department of Biomedical Engineering, School of Life Science and

Technology, Xi'an Jiaotong University, Xi'an, China

Full list of author information is available at the end of the article
}

two decades. At present, there are many types of CNV recognition methods based on various gene-sequencing methods. Among them are three typical methods: the fluorescence in situ hybridization (FISH), the highthroughput sequencing (HTS) and the array-based comparative genomic hybridization $(\mathrm{aCGH})$ [5]. FISH has a relatively low resolution, and only large repeats can be detected [6]. aCGH only has a high genomic resolution for large CNVs [7]. HTS has a high genomic resolution and can detect not only repetitions but also other types of structural variations in the genome [6]; hence, HTS has become the most popular method for gene sequencing in CNV detection. With the in-depth study of HTS, two branches of research have been developed-the whole genome sequencing (WGS) and the whole exome

(c) The Author(s). 2020 Open Access This article is distributed under the terms of the Creative Commons Attribution 4.0 International License (http://creativecommons.org/licenses/by/4.0/), which permits unrestricted use, distribution, and 
sequencing (WES). WGS is the laboratory process of determining most DNA base pairs across the 46 chromosomes of an individual's genome [8], and WES is a process of sequencing all protein-coding regions of genes in a genome [9]. Compared with WGS, WES was proposed later; however, it is more popular in clinical diagnostics and academic research, which due to two advantages of WES: (1) WES is an effective technique for the study of rare Mendelian and common polygenic diseases, such as Alzheimer's disease; (2) WES is cheaper than WGS. Thus, the CNV detection based on WES has become a research hotspot, and increasingly many have been conducted on methods for detecting CNVs from genes with WES data. However, although increasingly many $\mathrm{CNV}$ detection tools are becoming available, there is still no recommended reference for WES-based CNV detection tools in medical applications, which hinders the use of CNV detection tools in practice.

To resolve this problem, in our study, firstly, we chose four tools, which are usually appearing in the literature on $\mathrm{CNV}$ tool evaluation and are based on the read depth approach. Then, we comprehensively evaluated their performances in terms of the following three aspects: (1) the sensitivity and specificity of $\mathrm{CNV}$ detection for various coverages, $\mathrm{CNV}$ sizes and $\mathrm{CNV}$ types; (2) the overlapping consistency; (3) the computational cost. Finally, by comparing the performances of these tools, our study not only identifies the limitations and advantages of each CNV tool but also provides a recommended reference on $\mathrm{CNV}$ tools according to various requirements, which will facilitate researchers in the selection of suitable tools for their projects.

\section{Conclusions}

In this study, first, we selected four WES-based CNV detection tools: CoNIFER, cn.MOPS, CNVkit and exomeCopy. Then, we comprehensively evaluated and compared the performances of four selected CNV tools. Finally, by analyzing the experimental results, we recommend suitable CNV tools according to the application requirements, which are listed in Table 1. According to this table, we can divide the requirements into two categories: (1) The accuracy is the primary comparison criterion. (2) The

Table 1 The recommended tool for different requirements

\begin{tabular}{lll}
\hline Requirements & & Recommendation \\
\hline Accuracy first & CNV size is small $(<100 \mathrm{~kb})$ & CNVkit \\
& CNV size is large & cn.MOPS \\
& More insertion & CoNIFER \\
& More deletion & CNVkit \\
& No prior knowledge & cn.MOPS+CoNIFER \\
Others & Speed first & cn.MOPS \\
& Low- memory & CONIFER \\
\hline
\end{tabular}

accuracy is not the primary comparison criterion. In the first category, if the target CNV sizes are small, the recommendation is CNVkit; otherwise, the recommendation is cn.MOPS. If the $\mathrm{CNV}$ insertions are more frequent than the CNV deletions, the recommendation is CoNIFER; otherwise, CNVkit is a satisfactory choice. However, in practice, the information of the target CNVs is typically unknown, and the recommendation of our study is to use CoNIFER and cn.MOPS together. In the other category, if the researcher must deal with large WES data rapidly and desires a typical sensitivity, the recommendation is cn.MOPS, whereas if the researcher wants to detect CNVs on any computer, including a low-configuration computer, the recommendation is CoNIFER. Thus, in practice, we can recommend a proper $\mathrm{CNV}$ tool to researchers according to their requirements, which can maximize the accuracy of the test result. For instance, according to Rohrback's study [10], the size of a CNV that is in a neuron of the human brain is typically between $2 \mathrm{Mb}$ and $10 \mathrm{Mb}$, and to obtain an accurate result for its CNV detection, the recommended $\mathrm{CNV}$ tool is cn.MOPS.

\section{Method}

Data sets

In this study, we used simulated WES data and real WES data to evaluate the performances of CNV tools. For simulated data, the CNV size, the CNV number and the coverage are available to the researchers, while those of real data are unknown.

For the simulated data, we used hg38 as the reference genome, which can be downloaded from The National Center for Biotechnology Information (NCBI), https:// www.ncbi.nlm.nih.gov/. Then, we simulated the test genome by using the reference genome, and in this process, three assumptions were made: (1) The CNV size exceeds $1 \mathrm{~kb}$. (2) The coverage is $100 \mathrm{X}$. (3) The length of the reads is $50 \mathrm{bp}$. After obtaining the test genome and the reference genome, we used bowtie 2 and samtools with their default parameters to obtain the aligned BAM file, which can be used by CNV tools directly.

For the real data, we selected ten real samples from the exome example of CNVkit, which can be downloaded from GitHub, https://github.com/etal/cnvkit-examples/tree/master/exome. Then, to enable the use of these data with other selected CNV tools, we converted these samples from cnn files to GRange objects and S4 objects; in addition, we created files in which the reads per kilobase per million mapped reads (RPKM) are recorded. After these steps, we can obtain real data that can be used with these $\mathrm{CNV}$ tools directly.

\section{Tools' comparisons}

To facilitate researchers in the selection of suitable WESbased $\mathrm{CNV}$ tools according to their requirements, we 
selected representative tools and evaluated their performances in various cases. In this process, one of tool selection criteria is that they are based on the read depth approach for the detection of $\mathrm{CNVs}$, which assumes that the variation in the read depth is unbiased, random and normally distributed and that the deviation from the background document may signify the presence of a CNV [11-13]. The other selection criterion is the availability of the tools.

With these criteria, first, we identified candidate tools from previous studies on the evaluation of $\mathrm{CNV}$ detection tools [14-16], such as CoNIFER [17], exomeCopy [18], ExCopyDepth [14], CNVkit [19], cn.MOPS [20] and so on. Then, we attempted to download these candidate tools. In the process, because the resources of some tools were unavailable, we screened out several of the CNV tools, such as ExCopyDepth. Finally, we selected four tools as the final tools by comparing the weekly downloads of candidate tools, and information on them is presented in Table 2.

According to Table 2, our selected CNV tools are CoNIFER, exomeCopy, CNVkit and cn.MOPS. Among them, CoNIFER uses singular value decomposition (SVD) to eliminate capture biases between sample batches and to detect CNVs in exome data [17]. exomeCopy uses the hidden Markov model (HMM) to detect CNVs in exome data [18]. CNVkit uses both targeted reads and nonspecific captured off-target reads to infer the copy number evenly across the genome for the detection of CNVs in exome data [19]. cn.MOPS uses Bayesian inference to detect CNVs in the exome data [20]. Tools differ in terms of their methods, which typically causes their performances to differ among cases. In practice, while using these CNV tools, we need to adjust their parameters to realize their optimal performances in various cases. However, this workload is heavy, and the simulation of all cases is unrealistic. Therefore, we chose a compromise in our study and adjusted parameters of each of the tools by evaluating their comprehensive performances for simulated data for which the CNVs' sizes range from $1 \mathrm{~kb}$ to $10 \mathrm{Mb}$ randomly. Our recommended parameters for these CNV tools are as follows: For CoNIFER, we set $s v d$ to 1 . For
exomeCopy, we set relto to 0.01 , goto.cnv to 0.0001 and goto.normal to 0.01. For CNVkit, we set target-avg-size to $300 \mathrm{bp}$. For cn.MOPS, we set priorImpact to 20, min.width to 9, upperThreshold to 0.4 and lowerThreshold to the default. All the experimental results of parameter adjustment are shown in additional file 1.

\section{Comparison criteria}

To more comprehensively assess the tools, we evaluated these tools mainly in terms of the sensitivity and specificity, overlapping consistency and computational costs. The comparison criteria are described in detail as follows.

\section{Sensitivity and specificity}

In medicine, sensitivity and specificity are two statistical measures that are widely used to evaluate the performance of a binary classification test [21]. Sensitivity is the statistic that measures the proportion of positive results that are correctly identified as such, and specificity is the statistic that measures the proportion of negative results that are correctly identified as such [22]. As in other medical dichotomy experiments, in our study, we used sensitivity and specificity as criteria for the performance evaluation of these $\mathrm{CNV}$ tools. In the process of calculating the sensitivity and specificity, we used the exon as the minimum unit, and calculation formulas for the sensitivity and specificity are presented as follows. The sensitivity is calculated via eq. (1) and is defined as the true positive rate (TPR). The specificity is calculated via eq. (2) and is defined as the true negative rate (TNR).

$$
\begin{aligned}
& T P R=\frac{T P}{P}=\frac{T P}{T P+F N} \\
& T N R=\frac{T N}{N}=\frac{T N}{T N+F P}
\end{aligned}
$$

In these equations, there are six values: positive $(\mathrm{P})$, negative $(\mathrm{N})$, true positive (TP), true negative $(\mathrm{TN})$, false positive (FP) and false negative (FN). They are defined in Table 3. From this table, we can obtain not only the definitions of these values but also the relationships among

\begin{tabular}{|c|c|c|c|c|c|}
\hline Tools & Language & Input format & Methodology & Installation tutorial & Reference \\
\hline CONIFER & python & $\begin{array}{l}\text { BAM/RPKM、 } \\
\text { bed、RPKM }\end{array}$ & $\begin{array}{l}\text { Principal Component } \\
\text { Analysis }\end{array}$ & http://conifer.sourceforge.net/index.html & $\begin{array}{l}\text { Krumm. } \\
\text { et al.(2012) [17] }\end{array}$ \\
\hline exomeCopy & $\mathrm{R}$ & $\begin{array}{l}\text { BAM、 bed、 } \\
\text { Grange }\end{array}$ & $\begin{array}{l}\text { A hidden Markov } \\
\text { model which } \\
\text { uses positional } \\
\text { covariates }\end{array}$ & $\begin{array}{l}\text { http://www.bioconductor.org/packages/release/bioc/html/ } \\
\text { exomeCopy.html }\end{array}$ & $\begin{array}{l}\text { Love. } \\
\text { et al.(2011) [18] }\end{array}$ \\
\hline CNVkit & python & $\begin{array}{l}\text { BAM、 bed、 } \\
\text { cnn }\end{array}$ & $\begin{array}{l}\text { Principal Component } \\
\text { Analysis }\end{array}$ & https://cnvkit.readthedocs.io/en/stable/ & $\begin{array}{l}\text { Eric. } \\
\text { et al.(2016) [19] }\end{array}$ \\
\hline cn.MOPS & $\mathrm{R}$ & $\begin{array}{l}\text { BAM、 bed、 } \\
\text { Grange }\end{array}$ & Bayesian approach & http://www.bioinf.jku.at/software/cnmops/cnmops.html & $\begin{array}{l}\text { Klambauer. } \\
\text { et al.(2012) [20] }\end{array}$ \\
\hline
\end{tabular}

Table 2 Selected representative CNV calling methods 
Table 3 The definition of six values in sensitivity and specificity

\begin{tabular}{ll}
\hline Values & Definition \\
\hline P (Positive) & Number of exons which are within the real CNV \\
N (Negative) & Number of exons which are out of the real CNV \\
TP (True Positive) & $\begin{array}{l}\text { Number of exons which are within both the detected } \\
\text { CNV and the real CNV }\end{array}$ \\
TN (True Negative) & $\begin{array}{l}\text { Number of exons which are out of both the detected } \\
\text { CNV and the real CNV }\end{array}$ \\
FP (False Positive) & $\begin{array}{l}\text { Number of exons which are within the detected } \\
\text { CNV and out of the real CNV }\end{array}$ \\
FN (False Negative) & $\begin{array}{l}\text { Number of exons which are out of the detected } \\
\text { CNV and within the real CNV }\end{array}$ \\
\hline
\end{tabular}

them, such as TP plus FN equals $\mathrm{P}$ and TN plus FP equals $\mathrm{N}$.

\section{Overlapping consistency}

The overlapping consistency is a comparison method that is typically used in medicine. In our study, since groundtruth information for the real data is unavailable, we abandoned the strategy of evaluating the performances of these $\mathrm{CNV}$ tools by comparing their sensitivity and specificity values under various conditions; instead, we used the Venn diagram [23-25] to evaluate their performances on real data. During this process, since the differences in the sample types may affect the results of the overlap test, we conducted this test for not only real data but also simulated data, and used the result for simulated data as the reference for the real data. To facilitate the evaluation, we quantified the consistency among these four tools. Here, we introduce the overlap rate as the quantitative value, which is defined as the ratio between $\mathrm{N}_{-}$(overlap) and $\mathrm{N}$, where $\mathrm{N}$ is the total number of exons that are detected by a CNV tool and N_(overlap) is the number that overlapped with those that were detected by the other tools. For example, from Fig. 4a, for cn.MOPS, $N=6405$, and $\mathrm{N}_{\text {_}}$ (overlap) $=\mathrm{N}-58$; hence, the overlap rate is $99 \%$. This process aims at determining the consistency of these tools' results. If the overlap rates detected by these tools are high, the results of these tools have high CNV consistency and are trustworthy.

\section{Computational costs}

Computational costs are typically used to evaluate the performance of an algorithm in computer science. To make our assessment comprehensive, we assessed these $\mathrm{CNV}$ tools in terms of not only statistical characteristics but also computational costs. In the evaluation, computational costs include the time complexity and the space complexity. The time complexity refers to the computational effort that is required to execute the algorithm, which can be represented by the product of the central processing unit (CPU) utilization and the average running time. The space complexity is a measure of the size of the storage space that is required by the algorithm, which can be represented by the memory occupancy.

\section{Results \\ Sensitivity and specificity}

In our study, we used the sensitivity and the specificity to evaluate the performances of these selected tools. In this process, since the coverage, $\mathrm{CNV}$ size and $\mathrm{CNV}$ type of the WES data may influence the performances of tools, we simulated three types of data and studied the changes in the CNV tools' performances with respect to these three factors. The results are presented as follows.

\section{Coverage}

To evaluate the impact of the coverage on the CNV detection performances of these tools, we considered a series of WES datasets with coverages of 3X, 10X, 30X and $100 \mathrm{X}$ for which the probability of insertions is equal to the probability of deletions. Then, we used the selected tools to detect CNVs from these data. The results are presented in Fig. 1. Figure $1 \mathrm{a}$ and $\mathrm{b}$ presents the changes of these tools' sensitivities (TPRs) and specificities (TNRs) with respect to the coverage, and Fig. 1c presents the numbers of detected CNVs by these tools with various coverages.

From Fig. 1, we obtain three main conclusions: First, the sensitivity (TPR) increases rapidly and subsequently stabilizes with the increase of the data's coverage, which may be caused by the ceiling effect. Second, the specificity (TNR) decreases overall with the increase of the sensitivity. Finally, the number of detected CNVs of every tool increases initially and subsequently remains unchanged with the increase of the data's coverage. According to these results, the coverage of $100 \mathrm{X}$ is sufficient in practice, for which the sensitivities and specificities of these tools are satisfactory, and the computational burden is much lower than that for the data with higher coverage.

\section{CNV size}

To evaluate the influence of the CNV size on the CNV detection performance, we simulated a series of datasets as input, for which the $\mathrm{CNV}$ sizes are distributed in 1 $\mathrm{kb}-10 \mathrm{~kb}, 10 \mathrm{~kb}-100 \mathrm{~kb}, 100 \mathrm{~kb}-1 \mathrm{Mb}$ and $1 \mathrm{Mb}-10 \mathrm{Mb}$ while the coverage is $100 \mathrm{X}$ and each CNV type (deletion and insertion) occurs with equal frequency among them. Then, we used the selected tools to detect CNVs from these datasets. The results are presented in Fig. 2. Figure $2 \mathrm{a}$ and $\mathrm{b}$ show the changes of these tools' sensitivities (TPRs) and specificities (TNRs) with respect to the $\mathrm{CNV}$ size, and Fig. 2c shows the numbers of detected $\mathrm{CNV}$ s of various $\mathrm{CNV}$ sizes for these tools. For the abscissa axis of Fig. 2a and b, the CNV size* is a value that is computed from the CNV size by dividing the CNV size by 1000 , calculating the base 10 logarithm, and 


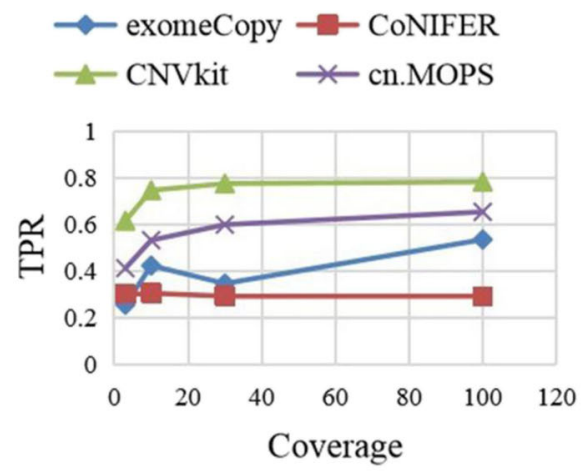

(a)
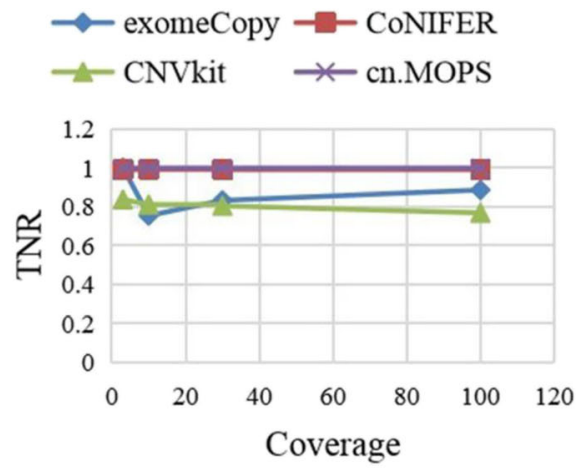

(b)

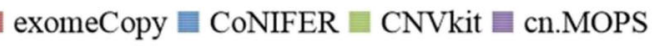

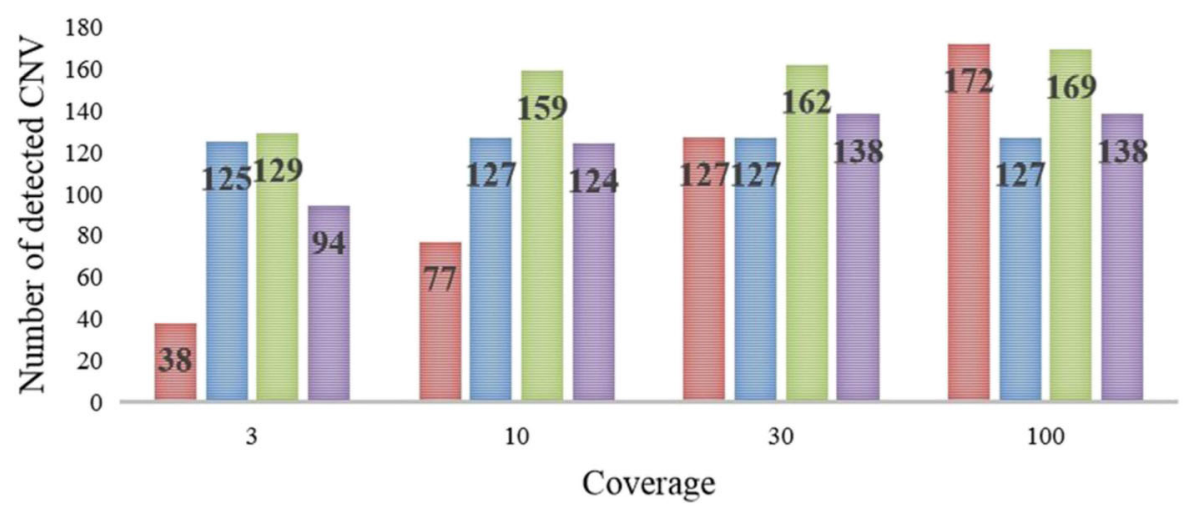

(c)

Fig. 1 The changes of tools' performances with respect to the coverage. Fig $\mathbf{a}$ and $\mathbf{b}$ describe the changes of these tools' sensitivities (TPRs) and specificities (TNRs) with respect to the coverage, and Fig $\mathbf{c}$ describes the numbers of detected CNVs in different coverages for these tools.

rounding up the value. For example, when the CNV size is $111 \mathrm{~kb}$, the CNV size* is 3 , and when the CNV size is $9 \mathrm{Mb}$, the CNV size* is 4 .

From Fig. 2, we draw two main conclusions: First, for all these tools, the sensitivity increases initially and subsequently remains unchanged or decreases slightly with the increase of the CNV size, while the specificity decreases as the sensitivity increases, and the number of detected CNVs increases as the CNV size increases. Second, the performances of these tools change with the CNV size, and the recommended tools differ among the cases. For example, when the targeted CNV size is between $1 \mathrm{~kb}$ and $100 \mathrm{~kb}$, CNVkit comprehensively outperforms other tools in terms of the sensitivity and specificity, whereas when the targeted CNV size is between $100 \mathrm{~kb}$ and $10 \mathrm{Mb}$, cn.MOPS performs best comprehensively in terms of the sensitivity and specificity.

After we obtained the sensitivities and specificities of these tools for various $\mathrm{CNV}$ sizes, since the targeted CNVs may be unknown, we calculated the global sensitivities and specificities of these tools by averaging their sensitivities and specificities over various CNV sizes. The results are presented in Table 4. According to the information in this table, cn.MOPS is a suitable choice for unknown research as its specificity and sensitivity are satisfactory comprehensively.

\section{CNV type}

To determine whether the CNV type influences the CNV detection or not, we simulated a series of datasets, of which the coverage is $100 \mathrm{X}$, the $\mathrm{CNV}$ size is random and the CNV types occur with equal frequency. Then, we used the selected tools to detect CNVs and counted the number of detected CNVs of each type. The results are presented in Fig. 3.

From Fig. 3, we conclude the following: First, all these tools can detect not only $\mathrm{CNV}$ insertions but also $\mathrm{CNV}$ deletions. Second, all tools except CoNIFER perform better for CNV deletions than for CNV insertions. Third, although CoNIFER performs better for insertions than for deletions, it may not perform the best among all these 


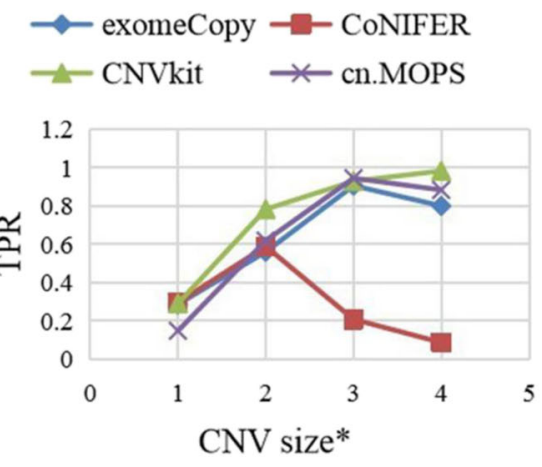

(a)
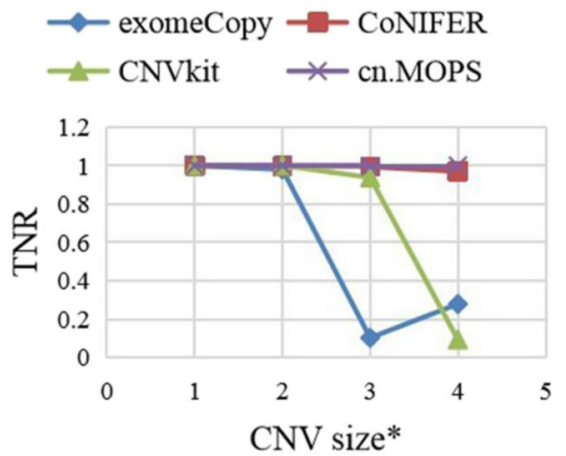

(b)

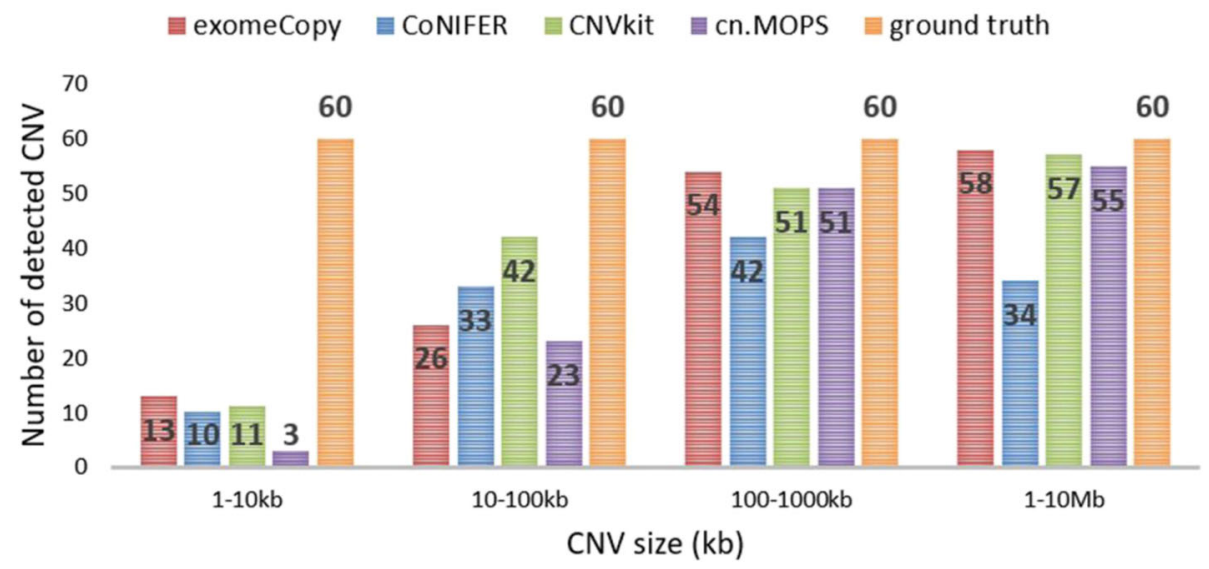

(c)

Fig. 2 The changes of tools' performances with respect to the CNV size. Fig a and $\mathbf{b}$ show the changes of these tools' sensitivities (TPRs) and specificities (TNRs) with respect to the CNV size, and Fig c shows the numbers of detected CNVs in different CNV sizes for these tools.

tools for insertions, of which the performance also depends on the distribution of the CNV size.

\section{Overlapping consistency}

In our study, to evaluate the consistency of these CNV tools, we conducted overlap tests on the simulated data and real data.

For the simulated data, first, we simulated a series of datasets, of which the coverage is $100 \mathrm{X}$ and the CNV size and type are random. Then, we used the selected four tools to detect CNVs. Finally, we drew a Venn diagram of the detection results, which is shown in Fig. 4a.

For the real data, first, we downloaded exome examples from CNVkit and used them as the original data. Then, we

Table 4 The global sensitivity and the global specificity of four CNV tools

\begin{tabular}{lllll}
\hline CNV detection tool & ExomeCopy & CoNIFER & CNVkit & cn.MOPS \\
\hline Sensitivity (TPR) & 0.64 & 0.29 & 0.75 & 0.65 \\
Specificity (TNR) & 0.60 & 0.99 & 0.76 & 1.00 \\
\hline
\end{tabular}

converted the original data (in cnn format) into the formats that are required by the other three CNV tools: RPKM format for CoNIFER, GRange format for exomeCopy and S4 for cn.MOPS. Finally, we detected CNVs and drew a Venn diagram by following the same procedure as for the simulated data. The Venn diagram is presented in Fig. 4b.

With the information in Fig. 4, we calculated the overlap rates (defined in section Comparison criteria) of these four tools to quantify their consistency, which are listed in Table 5.

According to Table 5, the overlap rates of CoNIFER, CNVkit and cn.MOPS exceed $90 \%$ for the simulated data; hence, they realize satisfactory consistency in the detection of CNVs, and their results are highly trustworthy. In addition, cn.MOPS and CoNIFER also realize satisfactory consistency (86 and 67\%) on the detection of CNVs from real data.

However, not all of these tools realize satisfactory consistency. The overlap rate of exomeCopy is always low ( $23 \%$ on simulated data and $3 \%$ on real data). To determine the cause of this phenomenon, we reviewed 


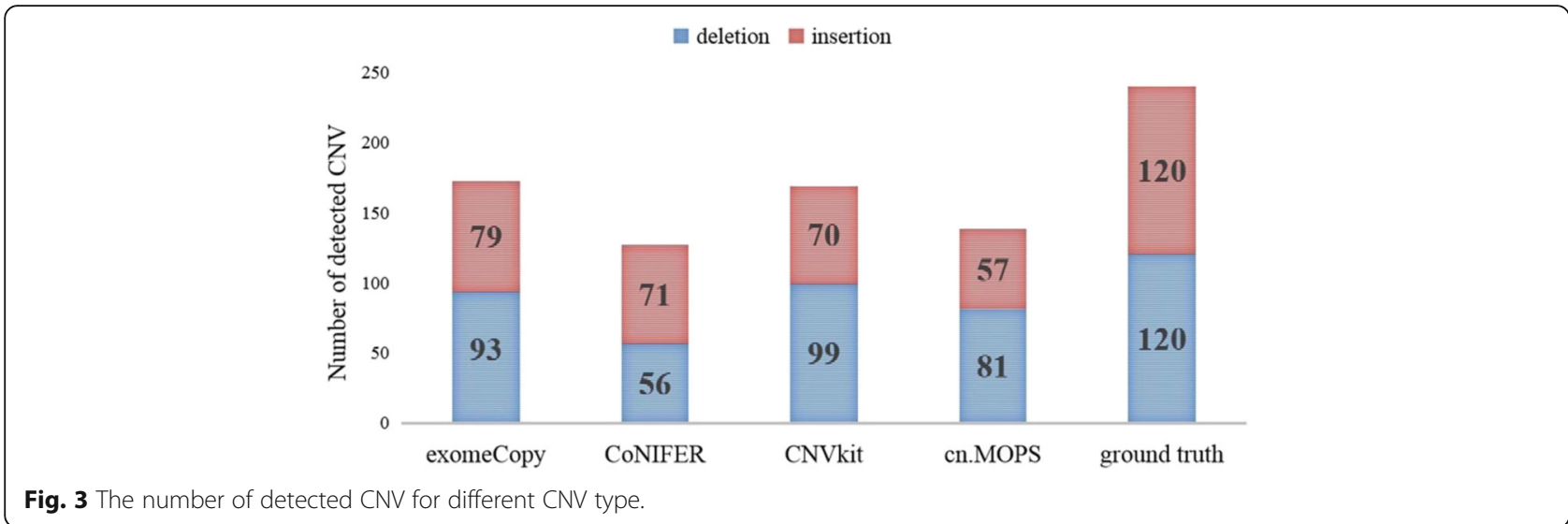

many other studies and found that our result is similar to Tan's results (22\%) [24], according to which the overlap rate of exomeCopy is associated with its algorithms.

In addition, we found that the tools' overlap rates for simulated data are higher than those for real data in our test. To determine what led to this phenomenon, we made the Venn diagrams of three of four tools, which were selected randomly. The results are presented in Fig. 5.

According to Fig. 5, all the combinations of three of these four tools have common exons except the combination of exomeCopy, cn.MOPS and CoNIFER, which is because the number of detected exons by cn.MOPS is too small relative to those by other tools. However, from the detection results on the simulated data, cn.MOPS outperformed most of the tools in terms of global sensitivity and specificity, which is not in line with the result of overlapping consistency. Based on the results from the simulated data, we think the underlying causes of this phenomenon may be that the CNV sizes of the samples don't focus on $10 \mathrm{~kb}$ to $1 \mathrm{Mb}$ and the number of $\mathrm{CNV}$ insertions exceeds the number of $\mathrm{CNV}$ deletions, which may cause the numbers of false detections for exomeCopy and CNVkit to be far larger than those for cn.MOPS and CoNIFER.

\section{Computational costs}

To assess these CNV tools comprehensively, we also used the computational cost as an evaluation criterion, which includes the time complexity and the space complexity. The results are presented as follows.

\section{Time complexity}

In our study, to determine the time complexities of these tools, we simulated a series of datasets as input, of which the coverage is $100 \mathrm{X}$ and the size is close to $11.2 \mathrm{MB}$. Then, since we do not have the detailed algorithm of these

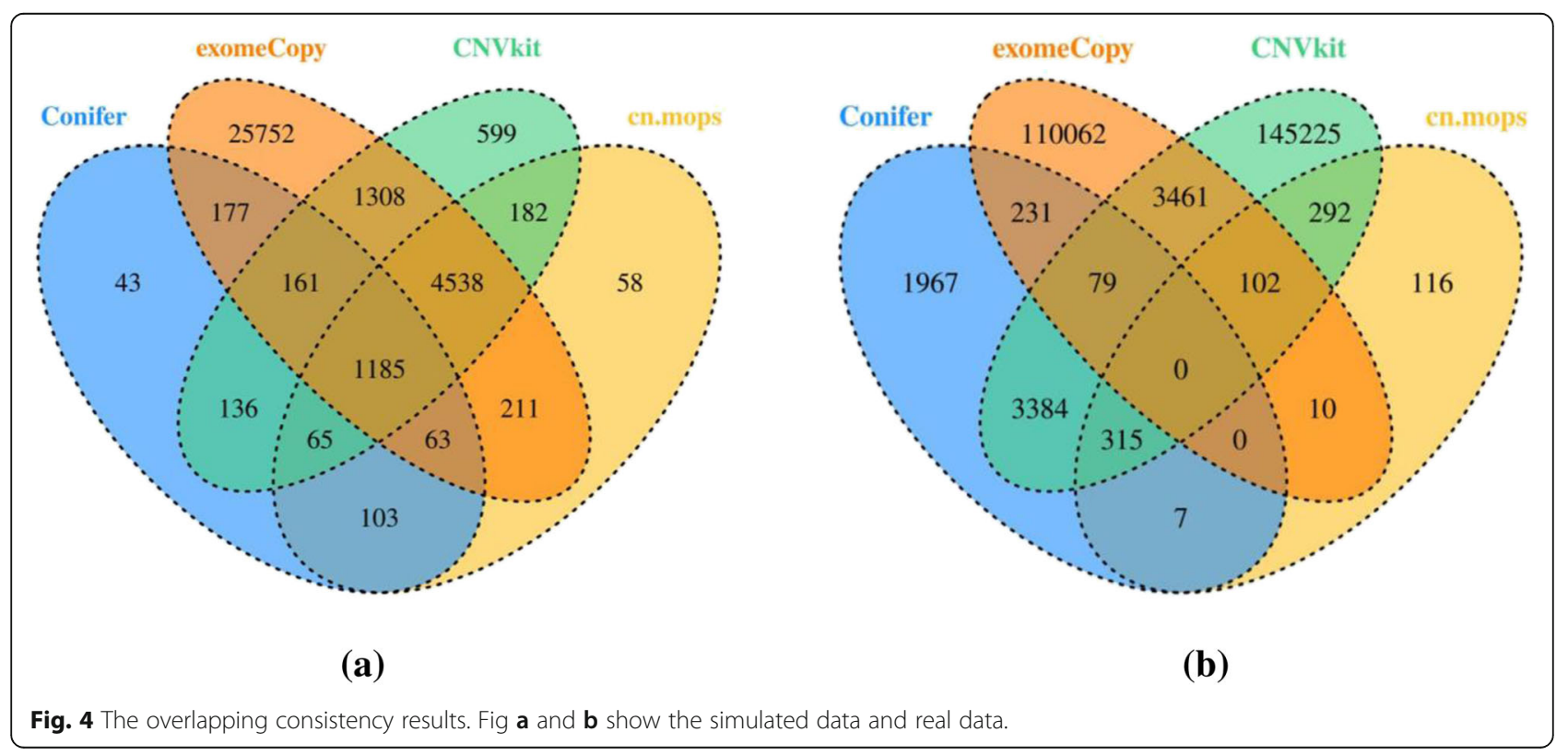


Table 5 The overlap rates of four CNV tools

\begin{tabular}{lllll}
\hline CNV detection tool & ExomeCopy & CoNIFER & CNVkit & cn.MOPS \\
\hline simulated data & 0.23 & 0.98 & 0.93 & 0.99 \\
real data & 0.03 & 0.67 & 0.05 & 0.86 \\
\hline
\end{tabular}

tools, we calculated the time complexity of each tool by multiplying the average running time and the CPU utilization. The results are presented in Fig. 6.

According to Fig. 6, cn.MOPS has the lowest time complexity; hence, it will require the minimum time for the same data processing among these tools. CNVkit has the highest time complexity, while it realizes satisfactory sensitivity and specificity.

\section{Space complexity}

To determine whether the CNV tool will affect other programs while it is running, we simulated a series of datasets as input, of which the coverage is $100 \mathrm{X}$ and the size is close to $11.2 \mathrm{MB}$. Then, we used the selected tools to detect CNVs from these datasets and calculated the average memory occupancy as a characterization quantity of the space complexity. The results are presented in Fig. 7.

According to the information in Fig. 7, CoNIFER has the lowest memory occupancy for the same data processing among these tools; hence, it has the minimum requirements for computer hardware. cn.MOPS has the highest memory occupancy among these tools, while it has the lowest time complexity, and this is because the time complexity and the space complexity are mutually constrained.

\section{Discussion}

With the development of CNV detection based on WES, increasingly many tools are being proposed and evaluated. However, no reference guide of $\mathrm{CNV}$ tools

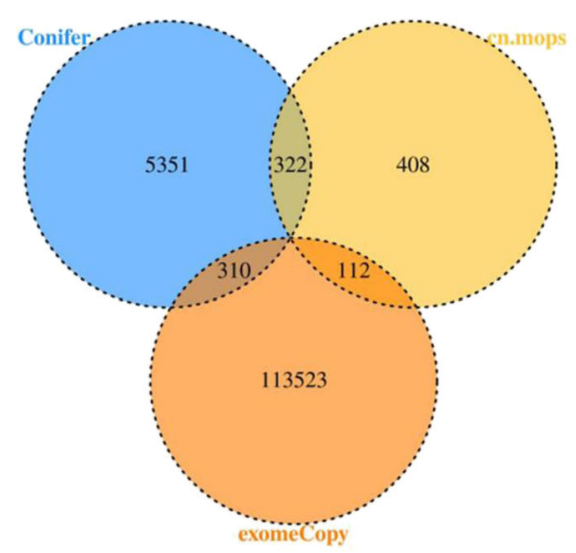

(a)

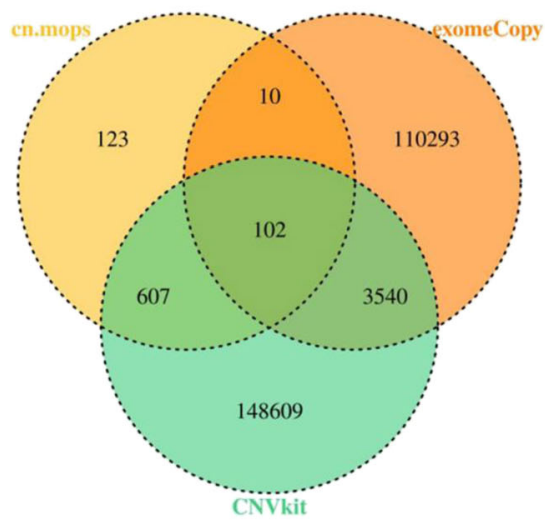

(c)

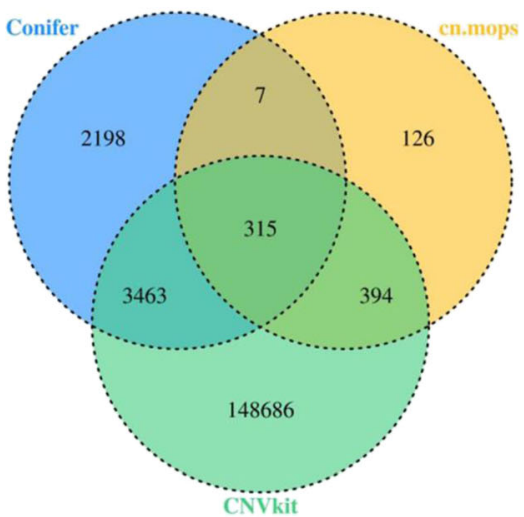

(b)

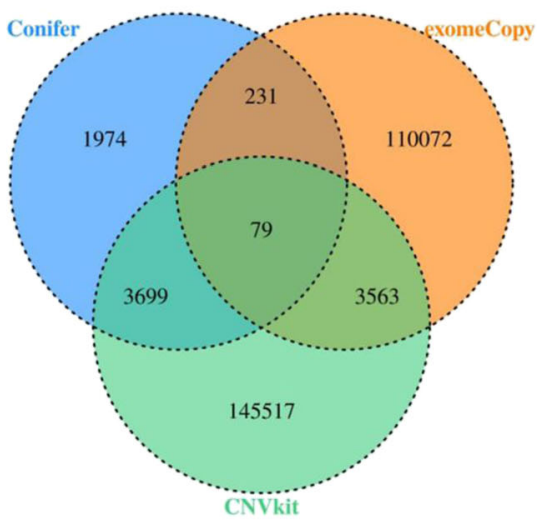

(d)

Fig. 5 Venn diagrams of three CNV tools. Fig a is for CoNIFER, cn.MOPS and exomeCopy, Fig b is for CoNIFER, cn.MOPS and CNVkit, Fig $\mathbf{c}$ is for CNVkit, cn.MOPS and exomeCopy, and Fig $\mathbf{d}$ is for CoNIFER, CNVkit and exomeCopy. 


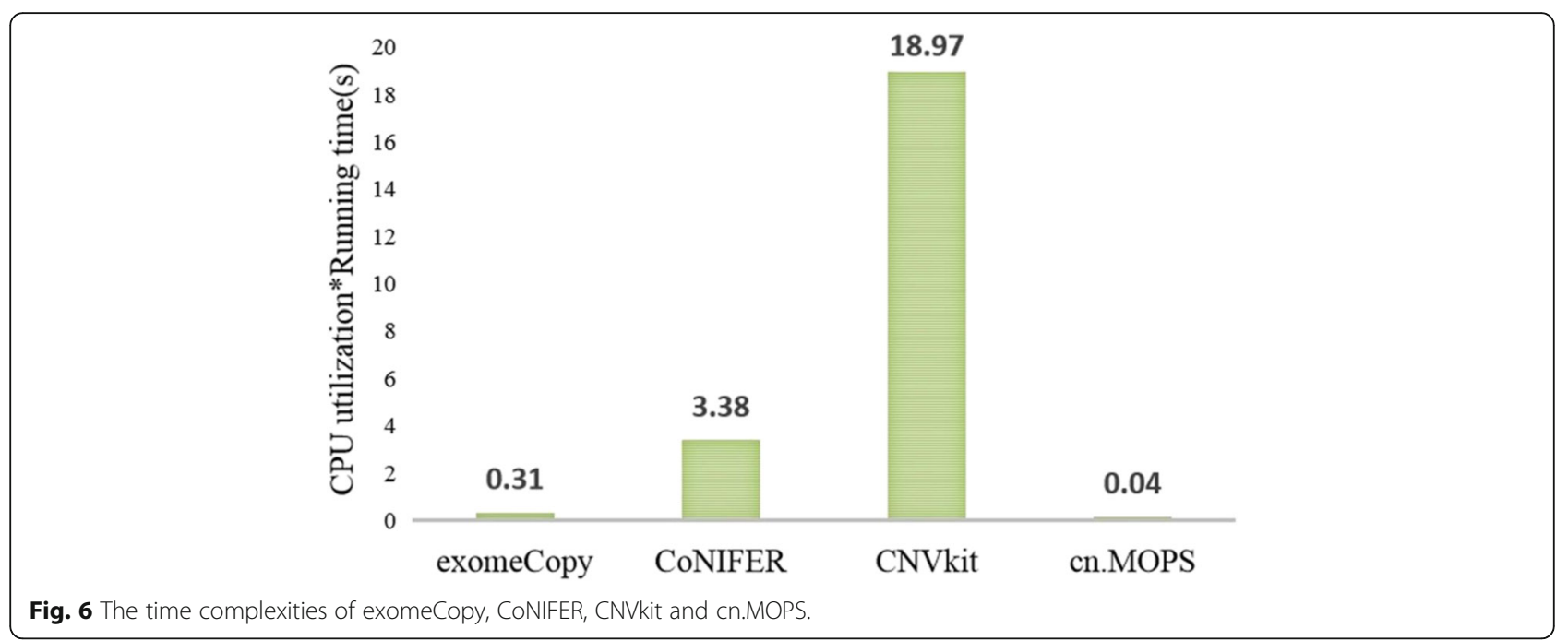

recommendation is available for various cases. Hence, the application of $\mathrm{CNV}$ tools in the clinic is challenging.

To facilitate clinicians and investigators in the selection of suitable CNV tools, we reviewed and compared prominent studies on evaluating CNV tools [23-25], and we identified three main areas for improvement: (1) the evaluation method. Most studies compared CNV tools only in terms of statistical characteristics, such as the sensitivity and specificity, and the influences of the coverage, CNV size and CNV type on tools' statistical characteristics were not considered. Therefore, in our study, we evaluated the performances of CNV tools in terms of not only statistical characteristics but also computational costs; in addition, we evaluated the influence of CNV characteristics; (2) the selection of CNV tools. In most studies, the latest tools were selected; however, the perfective maintenance stage in the software life cycle and user evaluations were not considered; therefore, in our study, we selected CNV tools according to the frequency of application, which is represented by the download frequency and the number of citations in the literature; (3) the practical guideline. Most studies identified advantages and disadvantages of tools without considering the users' background. Therefore, it is difficult for users to choose suitable tools for their projects. In our study, we recommend suitable CNV tools according to various conditions, which is helpful for users who have limited knowledge of computer science.

In the study, we have selected four representative WES-based tools and evaluated their performances from three aspects. From results of this study, we obtain information about the performances of these tools in various cases. Then, by comparing their performances, the most suitable tool can be identified in various scenarios, which will facilitate researchers in the selection of proper tools according to their projects. For example, among these tools, CNVkit has the highest sensitivity and a typical

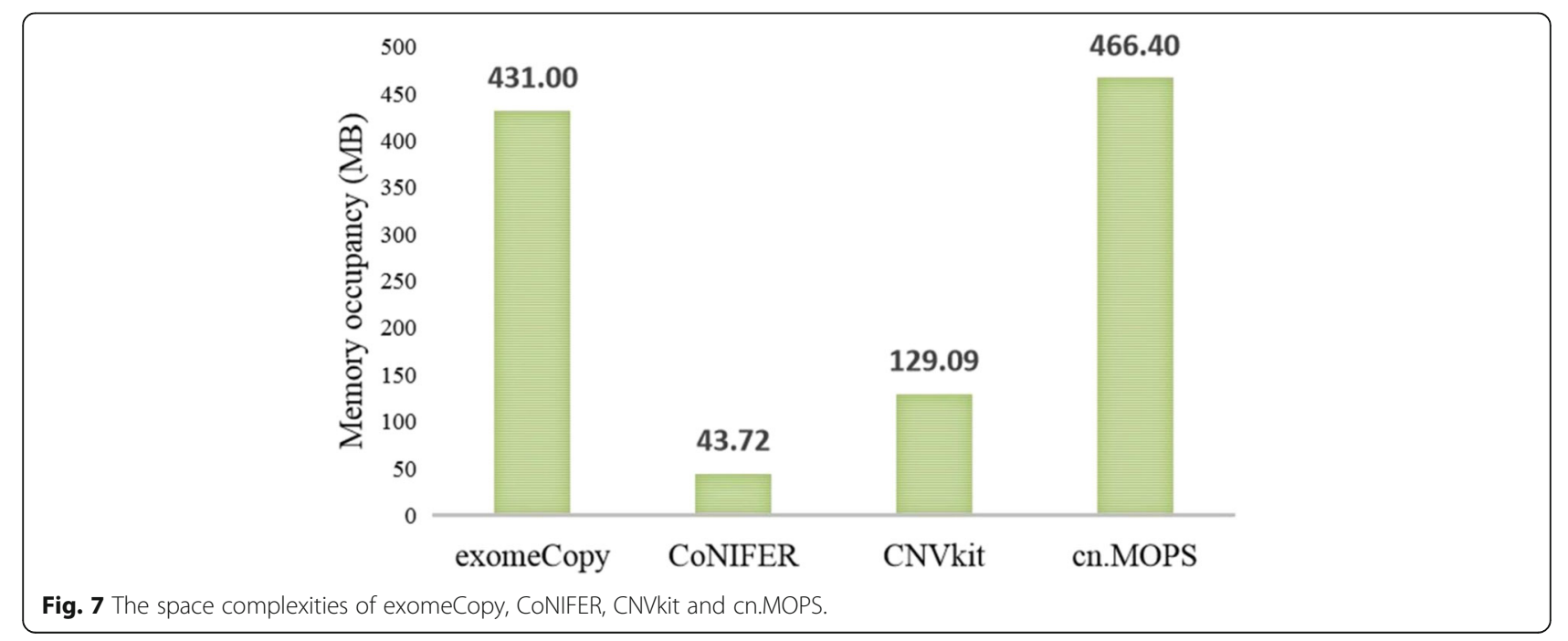


specificity for CNVs of which sizes are between $10 \mathrm{~kb}$ and $100 \mathrm{~kb}$; hence, CNVkit is the best choice for CNVs with sizes that are in this range.

Based on this study, we can recommend suitable CNV tools to researchers according to their projects, which can promote the clinical application of these tools. However, there are still many areas for improvement in this study: first, in this study, we selected only four WESbased tools as representatives for performance evaluation, and the guideline for $\mathrm{CNV}$ detection based on WES can be improved by adding more tools to this comparison. Second, the guideline is mainly for WES data with a coverage of approximately $100 \mathrm{X}$, and the performances of these tools for low coverage data are unknown in various cases. Finally, this study only evaluated the performances of WES-based CNV tools and ignored the comparison between WES-based CNV tools and WGS-based CNV tools.

\section{Supplementary information}

Supplementary information accompanies this paper at https://doi.org/10. 1186/s12859-020-3421-1.

Additional file 1: Selection of tool parameters. A text includes all the figures and tables about tool parameters' selection.

\section{Abbreviations}

aCGH: Array-based comparative genomic hybridization; CNV: Copy number variation; FISH: Fluorescence in situ hybridization; HMM: Hidden Markov model; HTS: High-throughput sequencing; kb: kilobase; Mb: Mbase; MB: MByte; NCBI: National Center for Biotechnology Information; RPKM: Reads per kilobase of exon model per million mapped reads; TNR: True negative rate; TPR: True positive rate; WES: Whole exome sequencing; WGS: Whole genome sequencing

\section{Acknowledgements}

Not applicable.

\section{Authors' contributions}

$L Z, X Y$ and JD designed the research, $H L$ and $K G$ created the dataset, $L Z$ and $\mathrm{HL}$ analyzed the data, LZ and JD wrote the manuscript. All authors read and approved the final manuscript.

\section{Funding}

This study was partially supported by National Science Foundation of China (Nos. 61771381 and 61571341)

\section{Availability of data and materials}

All data used in this study are available from the corresponding author upon request.

\section{Ethics approval and consent to participate}

Not applicable.

\section{Consent for publication}

Not applicable.

\section{Competing interests}

The authors declare that they have no competing interests.

\section{Author details}

${ }^{1}$ Department of Biomedical Engineering, School of Life Science and Technology, Xi'an Jiaotong University, Xi'an, China. ${ }^{2}$ School of Computer Science and Technology, Xidian University, Xi'an, China.
Received: 8 September 2019 Accepted: 17 February 2020

Published online: 05 March 2020

References

1. Mccarroll SA. Copy-number variation and association studies of human disease. Nat Genet. 2007;39:S37-42.

2. Stranger BE, Forrest MS, Dunning M, et al. Relative impact of nucleotide and copy number variation on gene expression phenotypes. Science. 2007; 315(5813):848-53

3. Hastings PJ, Lupski JR, Rosenberg SM, et al. Mechanisms of change in gene copy number. Nat Rev Genet. 2009;10(8):551-64.

4. Yuan X, Bai J, Zhang J, et al. CONDEL: detecting copy number variation and genotyping deletion Zygosity from single tumor samples using sequence data. IEEE/ACM Trans Comput Biol Bioinformatics. 2018. https://doi.org/10. 1109/TCBB.2018.2883333.

5. Duan J, Zhang JG, Deng HW, et al. CNV-TV: a robust method to discover copy number variation from short sequencing reads. BMC Bioinformatics. 2013;14(1):1-12

6. Freeman JL. Copy number variation: new insights in genome diversity. Genome Res. 2006:16(8):949-61.

7. Conrad B, Antonarakis SE. Gene duplication: a drive for phenotypic diversity and cause of human disease. Annu Rev Genomics Hum Genet. 2007:8(1):17-35.

8. Vassy JL, Lautenbach DM, Mclaughlin HM, et al. The MedSeq project: a randomized trial of integrating whole genome sequencing into clinical medicine. Trials. 2014;15(1):85.

9. $\mathrm{Ng}$ SB, Buckingham $\mathrm{KJ}$, Lee $\mathrm{C}$, et al. Exome sequencing identifies the cause of a mendelian disorder. Nat Genet. 2009;42(1):30-5.

10. Rohrback S, Siddoway B, Liu CS, et al. Genomic mosaicism in the developing and adult brain. Dev Neurobiol. 2018;78(11):1026-48.

11. Yoon S, Xuan Z, Makarov V, et al. Sensitive and accurate detection of copy number variants using read depth of coverage. Genome Res. 2009;19(9): 1586-92.

12. Kadalayil L, Rafiq S, Rosezerilli MJ, et al. Exome sequence read depth methods for identifying copy number changes. Brief Bioinform. 2014;16(3):883-6.

13. Sathirapongsasuti JF, Lee $\mathrm{H}$, Horst BAJ, et al. Exome sequencing-based copy-number variation and loss of heterozygosity detection: ExomeCNV. Bioinformatics. 2011:27(19):2648-54

14. Samarakoon PS, Sorte HS, et al. Identification of copy number variants from exome sequence data. BMC Genomics. 2014;15(1):661.

15. Wang H, Dan N, Ying K. Copy number variation detection using next generation sequencing read counts. BMC Bioinformatics. 2014;15(1):109.

16. Guo Y, Sheng Q, Samuels DC, et al. Comparative study of exome copy number variation estimation tools using Array comparative genomic hybridization as control. Biomed Res Int. 2013;2013(4):417-22.

17. Krumm N, Sudmant PH, Ko A, et al. Copy number variation detection and genotyping from exome sequence data. Genome Res. 2012;22(8):1525-32.

18. Love MI, Myšičková A, Sun R, et al. Modeling read counts for CNV detection in exome sequencing data. Stat Appl Genet Mol Biol. 2011;10(1):52.

19. Eric T, Hunter SA, Thomas B, et al. CNVkit: genome-wide copy number detection and visualization from targeted DNA sequencing. PLoS Comput Biol. 2016;12(4):e1004873.

20. Klambauer G, Schwarzbauer K, Mayr A, et al. cn.MOPS: mixture of Poissons for discovering copy number variations in next-generation sequencing data with a low false discovery rate. Nucleic Acids Res. 2012;40(9):e69.

21. Chatzimichail E, Paraskakis E, Rigas A. Predicting Asthma Outcome Using Partial Least Square Regression and Artificial Neural Networks: Hindawi Publishing Corp; 2013. https://doi.org/10.1155/2013/435321.

22. Watts N, Rani J, et al. Performance evaluation of improved skew detection and correction using FFT and median filtering. Int J Comput Appl. 2014:100:7-16.

23. Junbo D, Ji-Gang Z, Hong-Wen D, et al. Comparative Studies of Copy Number Variation Detection Methods for Next-Generation Sequencing Technologies. PLoS One. 2013;8(3):e59128.

24. Tan $R$, Wang $Y$, Kleinstein SE, et al. An evaluation of copy number variation detection tools from whole-exome sequencing data. Hum Mutat. 2014; 35(7):899-907.

25. Yao R, Zhang C, Yu T, et al. Evaluation of three read-depth based CNV detection tools using whole-exome sequencing data. Mol Cytogenet. 2017;10(1):30

\section{Publisher's Note}

Springer Nature remains neutral with regard to jurisdictional claims in published maps and institutional affiliations. 